\title{
Successful Resuscitation of Bone Cement Implantation Syndrome in a Patient with Pre-Existing Pulmonary Hypertension: A Case Report
}

\author{
Yeo S. H. Lynn'1, Kumaresh Venkatesan ${ }^{2}$ \\ ${ }^{1}$ Khoo Teck Puat Hospital/Woodlands Health Campus, Singapore \\ ${ }^{2}$ Khoo Teck Puat Hospital, Singapore \\ Email: lynn_yeo@whc.sg
}

How to cite this paper: Lynn, Y.S.H. and Venkatesan, K. (2020) Successful Resuscitation of Bone Cement Implantation Syndrome in a Patient with Pre-Existing Pulmonary Hypertension: A Case Report. Open Journal of Anesthesiology, 10, 293-297. https://doi.org/10.4236/ojanes.2020.108026

Received: July 11, 2020

Accepted: August 28, 2020

Published: August 31, 2020

Copyright $\odot 2020$ by author(s) and Scientific Research Publishing Inc. This work is licensed under the Creative Commons Attribution International License (CC BY 4.0).

http://creativecommons.org/licenses/by/4.0/

\section{(c) (i) Open Access}

\begin{abstract}
Background: In patients with pre-existing pulmonary hypertension undergoing surgery, there is an inherent risk of decompensation and right ventricular failure. Cemented hemi-arthroplasty in patients with pre-existing pulmonary hypertension predisposes them even more to morbidity and mortality from bone cement implantation syndrome (BCIS) with worsening of pulmonary hypertension. This risk should be recognized and steps taken for increased awareness, risk counselling and minimization of adverse effects. Case: We report a case of successful resuscitation of a patient with pre-existing pulmonary hypertension who developed 2 episodes of cardiac arrests-Grade 3 BCIS, shortly after cement implantation. Learning Points: Patients with pre-existing pulmonary hypertension for cemented hemi-arthroplasty are at additional risks and should be identified. Adequate risk counselling needs to be undertaken prior to surgery. A multi-disciplinary team effort is required. Discussion should be undertaken with the orthopaedic surgeon about the risks and benefits of using cemented implants. The anaesthetist needs to be vigilant for signs of BCIS, especially at the time of cement implantation and institute immediate resuscitation. Supportive treatment is the mainstay of management.
\end{abstract}

\section{Keywords}

Pulmonary Hypertension, Bone Cement Implantation Syndrome

\section{Introduction}

Bone cement implantation syndrome (BCIS) has been reported to be a poten- 
tially fatal complication in patients undergoing cemented hip arthroplasty. The pathophysiology has been previously investigated but no mechanism firmly proven. One hypothesis is the embolic model, in which emboli from increased intramedullary pressure lead to increase in pulmonary vascular resistance and right heart failure [1] [2]. Although there are many reports on BCIS, however not many focused on patients with pre-existing pulmonary hypertension. There is a study by Memtsoudis et al. [3] which showed that there is increased risk of perioperative mortality for patients with pulmonary hypertension undergoing major joint replacement.

We report a case of successful resuscitation of a patient with pre-existing pulmonary hypertension who developed 2 episodes of cardiac arrests-Grade 3 BCIS, shortly after cement implantation. It highlights the need to be aware of the patient's risk profile, vigilant monitoring and timely resuscitation.

\section{Case Report}

A 73-year-old lady presented to the hospital with a displaced left neck of femur fracture on the same day after a mechanical fall. Prior to the fall, she was able to mobilize with supports at home. Her past medical history included primary orthostatic hypotension, atrial fibrillation, moderate pulmonary hypertension, thalassemia trait and hyperlipidaemia. Her chronic medications were captopril, bisoprolol, esomeprazole, iron polymaltose, simvastatin, fludrocortisone, midodrine and aspirin. She had undergone L3/4 laminectomy for spinal stenosis in the past. 2D-Echocardiography (2DE) performed 2 weeks earlier revealed normal right and left ventricular systolic function, moderate pulmonary hypertension with estimated pulmonary artery systolic pressure (PASP) $58 \mathrm{mmHg}$ and moderately severe tricuspid valve regurgitation. She was planned for further review for pulmonary hypertension; however, she sustained the mechanical fall and was planned for a semi-urgent left hip bipolar hemiarthroplasty.

The following day, the patient underwent a left hip bipolar hemiarthroplasty under general anaesthesia. Anaesthesia was induced with intravenous (IV) fentanyl $100 \mathrm{mcg}$, IV propofol $100 \mathrm{mg}$ and IV atracurium $20 \mathrm{mg}$. Size 4 Proseal laryngeal mask airway was inserted and she was turned right lateral. Blood pressure was supported with intermittent boluses of IV phenylephrine (total 900 $\mathrm{mcg})$.

Ten minutes after bone cement implantation, the patient's oxygen saturation dropped from $98 \%$ to $91 \%$, accompanied with a drop in end-tidal carbon dioxide levels. Over the next few minutes, her plethysmographic trace was not detectable and her blood pressure had dropped to $53 / 28 \mathrm{mmHg}$. She progressed to pulseless electrical activity. Code Blue was activated. Surgery was aborted. She was turned supine and cardiopulmonary resuscitation was started immediately. She was given IV adrenaline boluses, $2.5 \mathrm{mg}$ in total. Return of spontaneous circulation (ROSC) was established within 3 minutes. IV noradrenaline infusion was started and she was transferred to the Intensive Care Unit (ICU). 
In ICU, she developed another episode of PEA and ROSC was achieved after 3 minutes of CPR and $1 \mathrm{mg}$ of IV adrenaline. She developed fast atrial fibrillation with rate of 150 to 175 beats per minute and was given bolus IV amiodarone $300 \mathrm{mg}$ and continued on an infusion of amiodarone for 24 hours. Blood pressure was supported with IV noradrenaline infusion, which was gradually weaned off.

A repeat 2DE the next day revealed good left ventricular function, worsening pulmonary hypertension with PASP $70 \mathrm{mmHg}$, severe tricuspid regurgitation, functional mild to moderate mitral regurgitation. There were no regional wall movement abnormalities. She regained consciousness with good neurological recovery and underwent left hip wash out and secondary closure, for completion of the initial surgery. Following this, she was extubated awake, remained hemodynamically stable and was discharged from ICU.

\section{Discussion}

BCIS is not uncommon, the total incidence estimated to be $28 \%$ [1], regardless of severity. A grading system has been proposed according to levels of desaturation and hypotension, of which Grade 3, cardiopulmonary arrest, which our patient had experienced, is considered the most severe form.

No mechanism has been firmly proven but one of the models proposed is the embolic model. [2] The postulated mechanism is that high intra-medullary pressures develop during cement implantation and prosthesis insertion causing a mechanical effect and release of mediators, leading to increased pulmonary hypertension.

Debris from the surgical site, including fat, cement particles, bone particles, platelets and fibrin cause obstruction at the right atrium, right ventricle and pulmonary artery intra-operatively. This has been seen on trans-esophageal echocardiography and described as a "snow flurry" [4]. Fat and marrow have also been shown in the lungs of patients who develop intra-operative deaths after cemented arthroplasty [1]. In addition to mechanical effects, it has been suggested that vasoactive or pro-inflammatory substances are released that increase pulmonary vascular resistance.

Clinical deterioration is likely to be caused by the increased pulmonary vascular resistance leading to acute right heart failure with reduced left ventricular filling and decrease in cardiac output. There is also decrease in coronary perfusion, leading to cardiac ischemia [5]. The end result is cardiac arrest with high risk of morbidity and mortality.

Bone cement has been used in hip hemiarthroplasty because of increased stability, less loosening of the prosthesis and lower re-operation rates. Studies have shown that the haemodynamic changes and embolic load were increased when bone cement was used compared to when cement was not used [6] [7]. It is plausible to suggest that it may be safer for bone cement not to be used in patients with pre-existing cardiopulmonary diseases. 
In a study by $M$. Hossain et al., the risk of perioperative death was increased following cemented implant insertion and the risk was exacerbated in patients with pre-existing cardiovascular morbidity [6].

In patients with pre-existing pulmonary hypertension undergoing surgery, there is an inherent risk of decompensation and right ventricular failure. G. Ramakrishna et al. has shown that, in patients with pulmonary hypertension undergoing elective non-cardiac surgery, the 30 -day morbidity was $42 \%$ and mortality 7\% [8]. Special care needs to be taken to ensure to avoid increases in pulmonary vascular resistance that can precipitate right ventricular failure by avoiding risk factors such as hypoxia, hypercarbia, acidosis, hypothermia and adequate management of pain.

Patients with pre-existing pulmonary hypertension are even more predisposed to morbidity and mortality from BCIS in view of the increased risk of worsening pulmonary hypertension and right heart failure [2] [9]. Memtsoudis et al. has shown that patients with pulmonary hypertension undergoing total hip arthroplasty has an approximately 4 -fold increase adjusted risk of mortality $(2.4 \%$ vs $0.6 \%$ ) compared to with patients without pulmonary hypertension in a matched sample [3].

These patients at additional risks should be identified and adequate risk counselling undertaken prior to surgery. A multi-disciplinary team effort is required. Discussion should be undertaken with the orthopaedic surgeon about the risks and benefits of using cemented implants. Safety guidelines for patients undergoing cemented hemiarthroplasty for hip fracture recommend that the patient should be well hydrated and blood pressure kept within $20 \%$ of pre-induction values. For patients at higher risk, invasive blood pressure monitoring should be carried out [10]. Although there have been cases of delayed presentation of BCIS [11], most of the time, it occurs during or shortly after time of cement implantation. Immediately prior to cement insertion, the anesthetist should be notified and on vigilance for signs of BCIS. There can be pre-defined resuscitative roles for staff present during cement implantation, to minimize confusion and delays in the event of a cardiac arrest. In addition, surgical techniques that help to lower the risk of BCIS should be used when appropriate.

The diagnosis of BCIS is supported by haemodynamic collapse and increase in PASP immediately following bone cement implantation. She developed another episode of cardiac arrest, showing that continued close monitoring is important. As previously reported, the management of BCIS is supportive and usually resolves within 24 to 48 hours [7]. Timely resuscitation and continued supportive therapy enabled our patient to recover.

\section{Conclusion}

Patients with pre-existing pulmonary hypertension are even more predisposed to morbidity and mortality from BCIS in view of the increased risk of worsening pulmonary hypertension and right heart failure. This risk should be recognized 
and steps taken for increased awareness, risk counselling and minimization of adverse effects.

\section{Acknowledgements}

Published with written consent of the patient.

\section{Conflicts of Interest}

The authors declare no conflicts of interest regarding the publication of this paper.

\section{References}

[1] Olsen, F., et al. (2014) Bone Cement Implantation Syndrome in Cemented Hemiarthroplasty for Femoral Neck Fracture: Incidence, Risk Factors, and Effect on Outcome. British Journal of Anaesthesia, 113, 800-806. https://doi.org/10.1093/bja/aeu226

[2] Donaldson, A.J., et al. (2009) Bone Cement Implantation Syndrome. British Journal of Anaesthesia, 102, 12-22. https://doi.org/10.1093/bja/aen328

[3] Memtsoudis, S.G., et al. (2010) Perioperative Mortality in Patients with Pulmonary Hypertension Undergoing Major Joint Replacement. Anesthesia \& Analgesia, 111, 1110-1116. https://doi.org/10.1213/ANE.0b013e3181f43149

[4] Lafont, N.D., et al. (1994) Embolism Detected by Transoesophageal Echocardiography during Hip Arthroplasty. Canadian Journal of Anesthesia, 41, 850-853. https://doi.org/10.1007/BF03011592

[5] Hines, C.B. (2018) Understanding Bone Cement Implantation Syndrome. AANA Journal, 86, 433-441.

[6] Hossain, M., et al. (2012) Is There a Difference in Perioperative Mortality between Cemented and Uncemented Implants in Hip Fracture Surgery? Injury, 43, 2161 2164. https://doi.org/10.1016/j.injury.2012.08.043

[7] Khanna, G., et al. (2012) Bone Cement and the Implications for Anaesthesia, Continuing Education in Anaesthesia. Critical Care \& Pain, 12, 213-216. https://doi.org/10.1093/bjaceaccp/mks011

[8] Ramakrishnan, G., et al. (2005) Impact of Pulmonary Hypertension on the Outcomes of Noncardiac Surgery: Predictors of Perioperative Morbidity and Mortality. Journal of the American College of Cardiology, 45, 1691-1699.

[9] Urban, M.K., et al. (2014) A Patient with Severe Pulmonary Hypertension for Arthroplasty. HSS Journal, 10, 171-179. https://doi.org/10.1007/s11420-014-9383-y

[10] Griffiths, R., et al. (2015) Safety Guideline: Reducing the Risk from Cemented Hemiarthroplasty for Hip Fracture. Anaesthesia, 70, 623-626. https://doi.org/10.1111/anae.13036

[11] Singh, V., et al. (2016) Bone Cement Implantation Syndrome: A Delayed Postoperative Presentation. Journal of Clinical Anesthesia, 31, 274-277. https://doi.org/10.1016/j.jclinane.2016.01.041 